\title{
Nuclear mass table in relativistic continuum Hartree-Bogoliubov theory
}

\author{
Youngman $\mathrm{Kim}^{1,2, a}$ \\ ${ }^{1}$ Rare Isotope Science Project, Institute for Basic Science, Daejeon 305-811, Republic of Korea \\ ${ }^{2}$ CDFT collaboration
}

\begin{abstract}
We present nuclear mass table in the framework of relativistic continuum Hartree-Bogoliubov (RCHB) theory which properly treats pairing correlations in the presence of the continuum. We study the nuclear mass table mainly with spherical symmetry using the density functional PC-PK1. We then briefly discuss on-going calculations for deformed nuclei with axial symmetry in the framework of deformed RCHB theory.
\end{abstract}

\section{Introduction}

The study of exotic isotopes is now a key element of nuclear physics with the world-wide development of rare isotope beam facilities; the Cooler Storage Ring (CSR) at the Heavy Ion Research Facility in Lanzhou (HIRFL) in the People's Republic of China [1, 2], the RIKEN Radioactive Ion Beam Factory (RIBF) in Japan [3], the Facility for Antiproton and Ion Research (FAIR) in Germany [4], the Second Generation System On-Line Production of Radioactive Ions (SPIRAL2) at GANIL in France [5], the Facility for Rare Isotope Beams (FRIB) in the USA [6], the Rare Isotope Science Project (RISP) in Korea [7], etc.

Along with such promising developments in experiments, it is essential to have rigorous theoretical methods to understand the exotic nuclei being located far from the valley of stability. The covariant density functional theory (CDFT) [8] is one of the successful approaches both for stable nuclei and rare isotopes [9-11]. Some important exemplary works include the consistent description of spin degrees of freedom $[9,12]$ and natural origin of the pseudospin symmetry [13-16].

In this study we calculate the properties of nuclei from $Z=8$ to $Z=120$ using a relativistic continuum HartreeBogoliubov (RCHB) theory, which offers a proper treatment of pairing correlations in the continuum, with the relativistic point-coupling density functional, PC-PK1 [17], aiming to develop a reliable nuclear mass model. $\mathrm{Nu}$ clear masses are one of the crucial inputs for nucleosynthesis calculations, especially for the rapid neutron capture process. We study the nuclear mass table mainly with spherical symmetry and briefly mention our calculations being under way for deformed nuclei with axial symmetry. Some early results of this work, from $\mathrm{O}$ to Ti, were reported in Ref. [18]; for nuclear region from $Z=8$ to $Z=22$ there are 234 experimentally measured nuclei and the root

\footnotetext{
a e-mail: ykim@ibs.re.kr
}

mean square (rms) deviation of our results from the experiments is about $2.23 \mathrm{MeV}$. The complete spherical mass table from our study will be presented in Ref. [19].

In Sec. 2, we briefly describe RCHB theory with the PC-PK1 functional. We present some preliminary results mostly with spherical symmetry in Sec. 3 and summarize this work in Sec. 4.

\section{Nuclear covariant energy density functional with point-coupling interactions}

We do a brief sketch on relativistic point-coupling models $[17,20,21]$ whose Lagrangian density has the following structure

$$
\mathcal{L}=\mathcal{L}^{\text {free }}+\mathcal{L}^{4 \mathrm{f}}+\mathcal{L}^{\text {hot }}+\mathcal{L}^{\text {der }}+\mathcal{L}^{\text {em }},
$$

where

$$
\mathcal{L}^{\text {free }}=\bar{\psi}(i \not \partial-m) \psi .
$$

The four-nucleon contact terms are given by

$$
\begin{aligned}
\mathcal{L}^{4 \mathrm{f}}= & -\frac{1}{2} \alpha_{S}(\bar{\psi} \psi)(\bar{\psi} \psi)-\frac{1}{2} \alpha_{V}\left(\bar{\psi} \gamma_{\mu} \psi\right)\left(\bar{\psi} \gamma^{\mu} \psi\right) \\
& -\frac{1}{2} \alpha_{T S}(\bar{\psi} \vec{\tau} \psi) \cdot(\bar{\psi} \vec{\tau} \psi)-\frac{1}{2} \alpha_{T V}\left(\bar{\psi} \vec{\tau} \gamma_{\mu} \psi\right) \cdot\left(\bar{\psi} \vec{\tau} \gamma^{\mu} \psi\right),
\end{aligned}
$$

and higher order ones are

$$
\mathcal{L}^{\text {hot }}=-\frac{1}{3} \beta_{S}(\bar{\psi} \psi)^{3}-\frac{1}{4} \gamma_{S}(\bar{\psi} \psi)^{4}-\frac{1}{4} \gamma_{V}\left[\left(\bar{\psi} \gamma_{\mu} \psi\right)\left(\bar{\psi} \gamma^{\mu} \psi\right)\right]^{2} .
$$

The gradient terms are also included to simulate the finite range nature of nuclear forces

$$
\begin{aligned}
\mathcal{L}^{\text {der }}= & -\frac{1}{2} \delta_{S} \partial_{\nu}(\bar{\psi} \psi) \partial^{v}(\bar{\psi} \psi)-\frac{1}{2} \delta_{V} \partial_{v}\left(\bar{\psi} \gamma_{\mu} \psi\right) \partial^{v}\left(\bar{\psi} \gamma^{\mu} \psi\right) \\
& -\frac{1}{2} \delta_{T S} \partial_{\nu}(\bar{\psi} \vec{\tau} \psi) \cdot \partial^{v}(\bar{\psi} \vec{\tau} \psi)-\frac{1}{2} \delta_{T V} \partial_{\nu}\left(\bar{\psi} \vec{\tau} \gamma_{\mu} \psi\right) \cdot \partial^{v}\left(\bar{\psi} \vec{\tau} \gamma^{\mu} \psi\right)
\end{aligned}
$$


Table 1. Parameters in PC-PK1 and pairing strength

\begin{tabular}{lcl}
\hline & Values & Dimension \\
\hline \hline$m_{n}$ & 939 & $\mathrm{MeV}$ \\
$m_{p}$ & 939 & $\mathrm{MeV}$ \\
$\alpha_{S}$ & $-3.96291 \times 10^{-4}$ & $\mathrm{MeV}^{-2}$ \\
$\beta_{S}$ & $8.6653 \times 10^{-1}$ & $\mathrm{MeV}^{-5}$ \\
$\gamma_{S}$ & $-3.80724 \times 10^{-17}$ & $\mathrm{MeV}^{-8}$ \\
$\delta_{S}$ & $-1.09108 \times 10^{-10}$ & $\mathrm{MeV}^{-4}$ \\
$\alpha_{V}$ & $2.6904 \times 10^{-4}$ & $\mathrm{MeV}^{-2}$ \\
$\gamma_{V}$ & $-3.64219 \times 10^{-18}$ & $\mathrm{MeV}^{-8}$ \\
$\delta_{V}$ & $-4.32619 \times 10^{-10}$ & $\mathrm{MeV}^{-4}$ \\
$\alpha_{T V}$ & $2.95018 \times 10^{-5}$ & $\mathrm{MeV}^{-2}$ \\
$\delta_{T V}$ & $-4.11112 \times 10^{-10}$ & $\mathrm{MeV}^{-4}$ \\
$V_{0}$ & 685 & $\mathrm{MeV} \mathrm{fm}^{3}$ \\
\hline
\end{tabular}

Finally, the electromagnetic interactions are described by

$$
\mathcal{L}^{\mathrm{em}}=-\frac{1}{4} F^{\mu v} F_{\mu \nu}-e \frac{1-\tau_{3}}{2} \bar{\psi} \not A \psi,
$$

where $A_{\mu}$ is the electromagnetic potential and $F^{\mu v}$ is its field tensor.

From these Lagrangian densities, we can obtain the RHB equation [11]

$$
\left(\begin{array}{cc}
h_{D}-\lambda & \Delta \\
-\Delta^{*} & -h_{D}^{*}+\lambda
\end{array}\right)\left(\begin{array}{c}
U_{k} \\
V_{k}
\end{array}\right)=E_{k}\left(\begin{array}{c}
U_{k} \\
V_{k}
\end{array}\right),
$$

where $\lambda$ is the Fermi energy and $E_{k}$ is the eigenenergy of the quasiparticle. The Dirac Hamiltonian $h_{D}$ is given by

$$
h_{D}=\vec{\alpha} \cdot \vec{p}+\beta[M+S(\vec{r})]+V(\vec{r}),
$$

where the scalar $S(\vec{r})$ and vector $V(\vec{r})$ potentials are

$$
\begin{aligned}
S(\vec{r})= & \alpha_{S} \rho_{S}+\beta_{S} \rho_{S}^{2}+\gamma_{S} \rho_{S}^{3}+\delta_{S} \Delta \rho_{S}, \\
V(\vec{r})= & \alpha_{V} \rho_{V}+\gamma_{V} \rho_{V}^{3}+\delta_{V} \Delta \rho_{V}+e A_{0} \\
& +\alpha_{T V} \tau_{3} \rho_{T V}+\delta_{T V} \tau_{3} \Delta \rho_{T V}
\end{aligned}
$$

The scalar, vector, and isospin densities are expressed as

$$
\begin{aligned}
& \rho_{S}(\vec{r})=\sum_{k} \bar{V}_{k}(\vec{r}) V_{k}(\vec{r}), \\
& \rho_{V}(\vec{r})=\sum_{k} V_{k}^{\dagger}(\vec{r}) V_{k}(\vec{r}), \\
& \rho_{T V}(\vec{r})=\sum_{k} V_{k}^{\dagger}(\vec{r}) \tau_{3} V_{k}(\vec{r}) .
\end{aligned}
$$

The pairing gap is given by

$$
\Delta_{k k^{\prime}}\left(\vec{r}, \vec{r}^{\prime}\right)=-\sum_{\tilde{k} \tilde{k}^{\prime}} V_{k k^{\prime}, \tilde{k} k^{\prime}}\left(\vec{r}, \vec{r}^{\prime}\right) \kappa_{k k^{\prime}}\left(\vec{r}, \vec{r}^{\prime}\right),
$$

where $\kappa=U^{*} V^{T}$ is the pairing tensor. For the particleparticle channel we use the following density-dependent zero-range pairing force,

$$
V^{p p}\left(\vec{r}_{1}, \vec{r}_{2}\right)=-V_{0} \delta\left(\vec{r}_{1}-\vec{r}_{2}\right) \frac{1}{4}\left(1-P^{\sigma}\right)\left(1-\frac{\rho\left(\vec{r}_{1}\right)}{\rho_{0}}\right),
$$

where $P^{\sigma}$ is the spin exchange operator and the factor $\rho_{0}=0.152 \mathrm{fm}^{-3}$.

For the particle-hole channel, we use the PC-PK1 parameter set [17] which is fitted to observables of 60 selected spherical nuclei, including the binding energies, charge radii, and empirical pairing gaps. Table 1 shows the masses of proton and neutron, coupling constants, and pairing strength used in this work.

The nucleon drip line in this work will be determined by the Fermi energy and by the nucleon separation energy. As usual, the one nucleon separation energy is defined by

$$
\begin{aligned}
& S_{n}(Z, N)=B(Z, N)-B(Z, N-1), \\
& S_{p}(Z, N)=B(Z, N)-B(Z-1, N),
\end{aligned}
$$

and two nucleon separation energy is

$$
\begin{aligned}
& S_{2 n}(Z, N)=B(Z, N)-B(Z, N-2), \\
& S_{2 p}(Z, N)=B(Z, N)-B(Z-2, N) .
\end{aligned}
$$

When it is said bound nuclei in this work, it means that the nucleon separation energy is positive and at the same time the Fermi energy is negative.

\section{Results}

In this section we present some preliminary results, obtained by solving the RHB equation in coordinate space, which provides a fully self-consistent treatment of the continuum and its coupling to bound states. For details on the method used in this work to solve the RHB equation numerically, one may consult Ref. [18].

We find that there are 9035 bound nuclei from $Z=8$ to $Z=120$; in this nuclear region there are 2231 measured nuclei and the rms deviation of our results from the experimental data is about $7.96 \mathrm{MeV}$. As the complete results from our study will be reported in Ref. [19], in this article we show a part of our mass table from $Z=52$ to $Z=66$ in Fig. 1 with the drip lines predicted by some other nuclear mass models; FRDM [22], TMA [23], HFB21 [24], and WS3 [25]. Here, different colors in the figure represent the relative binding energy differences $\left(E_{b}^{\mathrm{Exp}}-E_{b}^{\mathrm{Cal}}\right) / E_{b}^{\mathrm{Exp}}$.

Note that our early results for nuclear region from $Z=8$ to $Z=22$ were presented in Ref. [18]; from $\mathrm{O}$ to Ti isotopes 402 nuclei are predicted to be bound and for the $234 \mathrm{nu}-$ clei whose masses are measured in experiments, the rms deviation is about $2.23 \mathrm{MeV}$. As observed in Ref. [18], it is seen in Fig. 1 that the neutron drip lines predicted in this work are quite different from the ones compiled from the previous studies [22-25], i.e. the neutron drip line from this study is extended further to neutron-rich region than the others. In Table 2, we list the number of neutron rich nuclei which are predicted to be bound in this work but unbound in FRDM.

Before closing this section, we shortly comment on our study in progress for deformed nuclei. To investigate the effect of deformation on the nuclear mass table, we extend our spherical mass table to the one with axial symmetry. To this end, we work with the deformed relativistic Hartree-Bogoliubov theory in the continuum (DRHBc) 


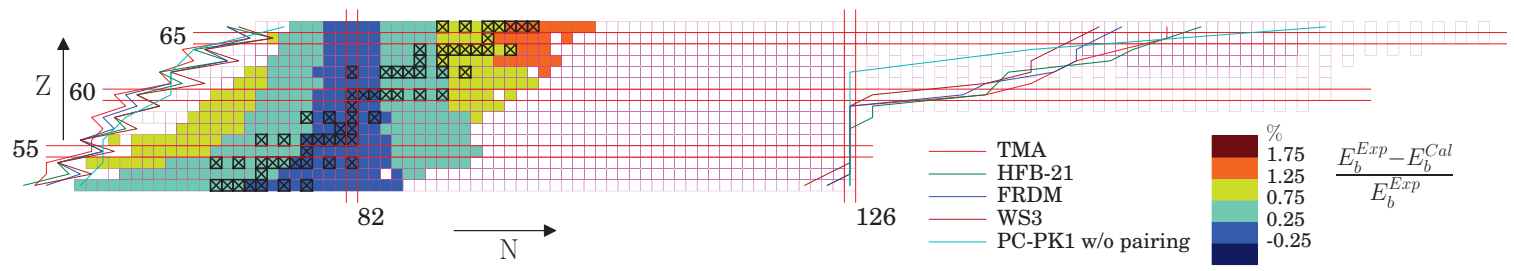

Figure 1. (Color online) Part of nuclear chart from Te to Gd predicted by RCHB theory with the functional PC-PK1. The relative binding energy differences $\left(E_{b}^{\text {Exp }}-E_{b}^{\text {cal }}\right) / E_{b}^{\text {Exp }}$ are marked with different colors. The nucleon drip lines predicted by some other mass models, FRDM [22], TMA [23], HFB21 [24], and WS3 [25], are also shown.

Table 2. $\mathcal{N}$ denotes the number of of neutron rich nuclei from $Z=22$ to $Z=66$ predicted to be bound in this work but unbound in FRDM.

\begin{tabular}{ll}
\hline Nuclei & $\mathcal{N}$ \\
\hline $\mathrm{Te}$ & 2 \\
$\mathrm{I}$ & 1 \\
$\mathrm{Xe}$ & 1 \\
$\mathrm{Pr}$ & 20 \\
$\mathrm{Nd}$ & 16 \\
$\mathrm{Pm}$ & 15 \\
$\mathrm{Sm}$ & 19 \\
$\mathrm{Eu}$ & 22 \\
$\mathrm{Gd}$ & 25 \\
$\mathrm{~Tb}$ & 27 \\
$\mathrm{Dy}$ & 25 \\
\hline
\end{tabular}

theory developed in Ref. [26], where the deformed RHB equations are solved in a Woods-Saxon basis to ensure a proper asymptotic behavior of the radial wave function. As an example of our results in the framework of the DRHBc, we show the binding energy of neon isotopes as a function of the mass number $A$ with two energy cutoff $E_{\text {cut }}$ values of Woods-Saxon basis in Fig. 2. It is seen in Fig. 2 that the calculated binding energies are mostly in agreement with those from experiments and the results are independent of $E_{\text {cut }}$.

\section{Summary}

We studied nuclear masses in the framework of RCHB theory, which properly treats pairing correlations in the presence of the continuum, mainly with spherical symmetry using the functional PC-PK1. We found that there are 9035 bound nuclei from $Z=8$ to $Z=120$; for nuclear region $Z=8$ to $Z=120$, there are 2231 measured nuclei and the rms deviation is about $7.96 \mathrm{MeV}$. Our study, RCHB with the density functional PC-PK1, predicted the wider neutron drip line than other nuclear mass models quoted in this work. This discrepancy might be due to pairing correlations in the continuum, functional (PC-PK1, relativistic), and spherical symmetry. We showed a part of our mass table from $Z=52$ to $Z=66$ in Fig. 1 with the nucleon drip lines predicted by some other nuclear mass models; FRDM [22], TMA [23], HFB21 [24], and WS3 [25]. The complete mass table will be reported in [19].

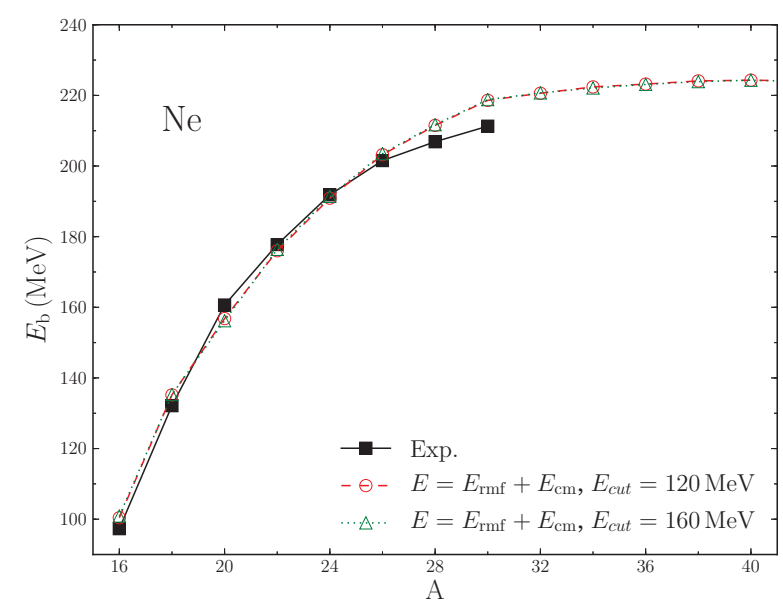

Figure 2. (Color online) Binding energies of Neon isotopes as a function of the mass number.

\section{Acknowledgements}

The author acknowledges the fruitful collaborations with Ying Chen, Haozhao Liang, Yeunhwan Lim, Jie Meng, Shuangquan Zhang, Xuewei Xia, and Shan-Gui Zhou. This work was supported in part by the Rare Isotope Science Project of Institute for Basic Science funded by Ministry of Science, ICT and Future Planning and National Research Foundation of Korea (2013M7A1A1075766).

\section{References}

[1] J.W. Xia, Nucl. Instrum. Meth. Phys. Res. A 488, 11 (2002)

[2] W. Zhan, H. Xu, G. Xiao, J. Xia, H. Zhao, and Y. Yuan, Nucl. Phys. A 834, 694c (2010)

[3] T. Motobayashi, Nucl. Phys. A 834, 707c (2010)

[4] C. Sturm, B. Sharkov and H. Stöcker, Nucl. Phys. A 834, 682c (2010)

[5] S. Gales, Nucl. Phys. A 834, 717c (2010)

[6] M. Thoennessen, Nucl. Phys. A 834, 688c (2010)

[7] http : //risp.ibs.re.kr/orginfo/info_blds.do [10]

[8] B.D. Serot and J.D. Walecka, Advances in Nuclear Physics, Vol. 16: The Relativistic Nuclear Many Body Problem (Plenum Press, New York, 1986) 
[9] P. Ring, Prog. Part. Nucl. Phys. 37, 193 (1996)

[10] D. Vretenar, A.V. Afanasjev, G.A. Lalazissis, and P. Ring, Phys. Rep. 409, 101 (2005)

[11] J. Meng, H. Toki, S.-G. Zhou, S.Q. Zhang, W.H. Long, and L.S. Geng, Prog. Part. Nucl. Phys. 57, 470 (2006)

[12] S.-G. Zhou, J. Meng, and P. Ring, Phys. Rev. Lett. 91, 262501 (2003)

[13] J. Meng, K. Sugawara-Tanabe, S. Yamaji, P. Ring, and A. Arima, Phys. Rev. C 58, R628 (1998)

[14] J. Meng, K. Sugawara-Tanabe, S. Yamaji, and A. Arima, Phys. Rev. C 59, 154 (1999)

[15] Chen Ti-Sheng, Lu Hong-Feng, Meng Jie, Zhang Shuang-Quan, and Zhou Shan-Gui, Chinese Phys. Lett. 20, 358 (2003)

[16] H. Liang, J. Meng, and S.-G. Zhou, Phys. Rep. 570, 1 (2015)

[17] P. W. Zhao, Z. P. Li, J. M. Yao and J. Meng, Phys. Rev. C 82, 054319 (2010)
[18] X. Qu, Y. Chen, S. Zhang, P. Zhao, I.J. Shin, Y. Lim, Y. Kim, and J. Meng, Sci. China Phys., Mech. \& Astron. 56, 2031 (2013)

[19] Xuewei Xia, et al., in preparation

[20] B.A. Nikolaus, T. Hoch, and D.G. Madland, Phys. Rev. C 46, 1757 (1992)

[21] T. Burvenich, D.G. Madland, J.A. Maruhn, and P.G. Reinhard, Phys. Rev. C 65, 044308 (2002)

[22] P. Möller, J.R. Nix, W.D. Myers and W.J. Swiatecki, Atom. Data Nucl. Data Tables 59, 185 (1995)

[23] L.S. Geng, H. Toki, and J. Meng, Prog. Theor. Phys. 113, 785 (2005)

[24] S. Goriely, N. Chamel, and J. M. Pearson, Phys. Rev. C 82, 035804 (2010)

[25] M. Liu, N. Wang, Y. Deng, and X. Wu, Phys. Rev. C 84, 014333 (2011)

[26] L. Li, J. Meng, P. Ring, E. G. Zhao, and S. G. Zhou, Phys. Rev. C 85, 024312 (2012) 